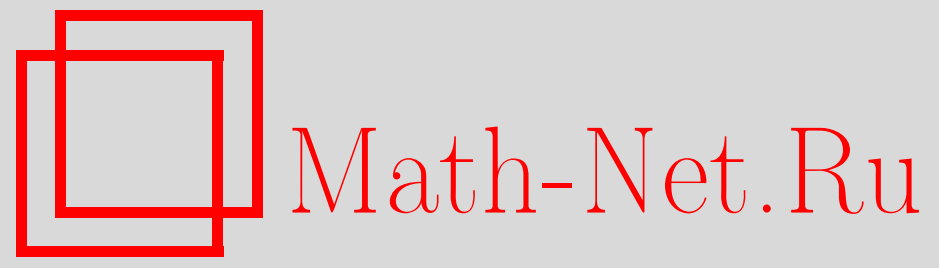

А. В. Колчин, Предельные теоремы для обобщенной схемы размещения, Дискрет. матем., 2003, том 15, выпуск 4, 148-157

DOI: https://doi.org/10.4213/dm224

Использование Общероссийского математического портала Math-Net.Ru подразумевает, что вы прочитали и согласны с пользовательским соглашением http://www . mathnet.ru/rus/agreement

Параметры загрузки:

IP : 34.229 .45 .116

26 апреля 2023 г., 18:36:43 


\title{
Предельные теоремы для обобщенной схемы размещения
}

\author{
() 2003 г. А. В. Колчин
}

\begin{abstract}
Для сумм независимых одинаково распределенных неотрицательных целочисленных случайных величин получен ряд предельных теорем, которые могут найти применение в задачах вероятностной комбинаторики при использовании обобщенной схемы размещения.

Работа поддержана грантом НШ 1758.2003.1 Президента Российской Федерации для поддержки ведущих научных школ Российской Федерации.
\end{abstract}

\section{1. Введение}

В вероятностной комбинаторике получил распространение подход, основанный на применении обобщенной схемы размещения, позволяющий сводить ряд комбинаторных задач к задачам о суммах независимых случайных величин, классическому объекту изучения в теории вероятностей $[1-4,6,13,14]$ Обобщенная схема размещения была введена в [1] и заняла заметное место в асимптотических исследованиях в вероятностной комбинаторике. Свое название эта схема получила в связи с тем, что она является обобщением классической задачи о случайном размещении частиц по ячейкам [2].

В обобщенной схеме размещения частиц распределение заполнений ячеек представимо как условное распределение независимых случайных величин при условии, что их сумма принимает фиксированное значение. Пусть $\eta_{1}, \ldots, \eta_{N}$ - неотрицательные целочисленные случайные величины, рассматриваемые как некоторые числовые характеристики комбинаторной структуры с $n$ элементами, состоящей из $N$ компонент, такие, что $\eta_{1}+\ldots+\eta_{N}=n$. Если существуют независимые случайные величины $\xi_{1}, \ldots, \xi_{N}$ такие, что совместное распределение $\eta_{1}, \ldots, \eta_{N}$ может быть представлено в виде

$$
\mathbf{P}\left\{\eta_{1}=k_{1}, \ldots, \eta_{N}=k_{N}\right\}=\mathbf{P}\left\{\xi_{1}=k_{1}, \ldots, \xi_{N}=k_{N} \mid \xi_{1}+\ldots+\xi_{N}=n\right\},
$$

где $k_{1}, \ldots, k_{N}$ - произвольные целые числа, то говорят, что $\eta_{1}, \ldots, \eta_{N}$ образуют обобщенную схему размещения с параметрами $n$ и $N$ и независимыми случайными величинами $\xi_{1}, \ldots, \xi_{N}$.

В силу независимости случайных величин $\xi_{1}, \ldots, \xi_{N}$ изучение многих характеристик обобщенной схемы размещения сводится к задачам о суммах независимых случайных величин. Пусть случайные величины $\xi_{1}, \ldots, \xi_{N}$ одинаково распределены и $\mu_{r}$ - число случайных величин $\eta_{1}, \ldots, \eta_{N}$, принявших значение $r, r=0,1, \ldots, N$. Нетрудно 
показать (см., например, [2]), что

$$
\mathbf{P}\left\{\mu_{r}=k\right\}=\left(\begin{array}{l}
N \\
k
\end{array}\right) p_{r}^{k}\left(1-p_{r}\right)^{N-k} \frac{\mathbf{P}\left\{\xi_{1}^{(r)}+\ldots+\xi_{N-k}^{(r)}=n-k r\right\}}{\mathbf{P}\left\{\xi_{1}+\ldots+\xi_{N}=n\right\}},
$$

где $\xi_{1}^{(r)}, \ldots, \xi_{N-k}^{(r)}$ - независимые одинаково распределенные случайные величины,

$$
\mathbf{P}\left\{\xi_{1}^{(r)}=k\right\}=\mathbf{P}\left\{\xi_{1}=k \mid \xi_{1} \neq r\right\}, \quad k=0,1,2, \ldots,
$$

и

$$
p_{r}=\mathbf{P}\left\{\xi_{1}=r\right\}, \quad r=0,1,2, \ldots
$$

Таким образом, получение предельного распределения случайной величины $\mu_{r}$ сводится к применению локальных теорем для сумм независимых случайных величин. В случае, когда распределения слагаемых одинаковы и фиксированы (не зависят от числа слагаемых), можно пользоваться хорошо развитой теорией суммирования независимых случайных величин [15]. Однако во многих применениях обобщенной схемы возникает необходимость в локальных предельных теоремах в схеме серий. В таких случаях до сих пор нет исчерпывающего ответа на вопрос, справедлива ли локальная предельная теорема даже в случае сходимости к нормальному закону, для каждого конкретного случая приходится проводить отдельное доказательство, либо следуя доказательству локальной теоремы, предложенному Б. В. Гнеденко $[15,1-7,9]$, либо проверяя условия общих локальных теорем $[10,8]$.

Обычно (см., например, [3]), распределение случайных величин $\xi_{1}, \ldots, \xi_{N}$ имеет вид

$$
\mathbf{P}\left\{\xi_{1}=k\right\}=\frac{b_{k} \theta^{k}}{k ! B(\theta)},
$$

где $b_{0}, b_{1}, b_{2}, \ldots$ - некоторая последовательность неотрицательных чисел,

$$
B(\theta)=\sum_{k=0}^{\infty} \frac{b_{k} \theta^{k}}{k !}
$$

и $\theta$ - параметр, принимающий положительные значение из области сходимости ряда $B(\theta)$. В таких случаях, если соотношение (1) справедливо при некотором значении $\theta$, то оно остается верным при всех положительных $\theta$ из области сходимости ряда $B(\theta)$ (см., например, [2-4]).

Для полного изучения характеристик обобщенной схемы размещения, как правило, требуются локальные предельные теоремы при всех значениях параметра $\theta$. В статье рассматриваются простейшие случаи. Во-первых, предполагается, что $b_{0}$ и $b_{1}$ положительны, и во-вторых, допускаются значения параметра $\theta$, строго меньшие радиуса сходимости ряда $B(\theta)$. Получены предельные теоремы о сходимости к нормальному распределению и распределению Пуассона. Остается не рассмотренным важный случай, когда значения параметра $\theta$ приближаются к границе сходимости ряда $B(\theta)$. Как показывают примеры (см., например, $[3,4,11,12])$, в этом случае могут появляться и другие предельные распределения.

Некоторые из доказанных в статье теорем, по-видимому, могут быть получены путем проверки приведенных в [10] общих условий справедливости локальных теорем о сходимости к нормальному распределению (см. [8]), однако автор считает, что наличие простых прямых доказательств может оказаться полезным для дальнейших исследований в этой области. 


\section{2. Основные обозначения. Вспомогательные соотношения}

Мы будем придерживаться следующих обозначений. Пусть задана последовательность неотрицательных чисел $b_{0}, b_{1}, b_{2}, \ldots$, таких, что $b_{0}, b_{1}>0$ и радиус сходимости $R$ ряда

$$
B(\theta)=\sum_{k=0}^{\infty} \frac{b_{k} \theta^{k}}{k !}
$$

положителен. Введем целочисленную случайную величину $\xi=\xi(\theta)$, распределенную по следующему закону:

$$
\mathbf{P}\{\xi=k\}=\frac{b_{k} \theta^{k}}{k ! B(\theta)}, \quad k=0,1,2, \ldots
$$

Положим

$$
m=m(\theta)=\mathbf{M} \xi, \quad \sigma^{2}=\sigma^{2}(\theta)=\mathbf{D} \xi, \quad \beta=\beta(\theta)=\mathbf{M}|\xi-m|^{3} .
$$

Заметим, что при $|\theta|<R$ случайная величина $\xi$ имеет все моменты.

Пусть $\xi_{1}, \xi_{2}, \ldots$ - независимые случайные величины, распределение которых совпадает с распределением (2) случайной величины $\xi$.

Нашей целью является получение предельных теорем для сумм вида

$$
S_{N}=\sum_{k=1}^{N} \xi_{k}
$$

Обозначим через $F_{N}(x)$ функцию распределения суммы центрированных и нормированных случайных величин

$$
S_{N}^{*}=\sum_{k=1}^{N} \frac{\xi_{k}-m}{\sigma \sqrt{N}} .
$$

Легко видеть, что производящая функция распределения случайной величины $\xi$ есть

$$
\phi(z)=\sum_{k=0}^{\infty} \frac{b_{k} \theta^{k} z^{k}}{k ! B(\theta)}=\frac{1}{B(\theta)} \sum_{k=0}^{\infty} \frac{b_{k}(z \theta)^{k}}{k !}=\frac{B(z \theta)}{B(\theta)} .
$$

Характеристическая функщия $\varphi(t)$ случайной величины $\xi$ есть $\phi\left(e^{i t}\right)$, поэтому

$$
\varphi(t)=\frac{B\left(\theta e^{i t}\right)}{B(\theta)} .
$$

Характеристическая функция $\varphi^{*}(t)$ центрированной и нормированной случайной величины $(\xi-m) /(\sigma \sqrt{N})$ есть $\phi\left(e^{i t /(\sigma \sqrt{N})}\right) e^{-i t m /(\sigma \sqrt{N})} ;$ поэтому

$$
\varphi^{*}(t)=\frac{B\left(\theta e^{i t /(\sigma \sqrt{N})}\right)}{B(\theta)} e^{-i t m /(\sigma \sqrt{N})} .
$$


Через $\bar{\varphi}(t)=\mathbf{M} e^{i t(\xi-m)}$ обозначим характеристическую функцию центрированной случайной величины $\xi-m$.

Выразим первые два момента случайной величины $\xi$ через функцию $B(\theta)$. Легко видеть, что

$$
\begin{aligned}
m=\mathbf{M} \xi & =\sum_{k=1}^{\infty} \frac{k b_{k} \theta^{k}}{k ! B(\theta)}=\frac{\theta B^{\prime}(\theta)}{B(\theta)} \\
\mathbf{M} \xi(\xi-1) & =\sum_{k=2}^{\infty} \frac{k(k-1) b_{k} \theta^{k}}{k ! B(\theta)}=\frac{\theta^{2} B^{\prime \prime}(\theta)}{B(\theta)} \\
\sigma^{2}=\mathbf{D} \xi & =\mathbf{M} \xi(\xi-1)+\mathbf{M} \xi-(\mathbf{M} \xi)^{2} \\
& =\frac{\theta^{2} B^{\prime \prime}(\theta)}{B(\theta)}+\frac{\theta B^{\prime}(\theta)}{B(\theta)}-\frac{\theta^{2}\left(B^{\prime}(\theta)\right)^{2}}{(B(\theta))^{2}}
\end{aligned}
$$

Введем также обозначение

$$
P_{N}(n)=\mathbf{P}\left\{S_{N}=n\right\}
$$

\section{3. Интегральные предельные теоремы}

Теорема 1. Пусть $N \rightarrow \infty$ и существуют постоянные $\theta_{0}, \theta_{1}, 0<\theta_{0}<\theta_{1}<R$, такие, что параметр $\theta=\theta(N) \in\left[\theta_{0}, \theta_{1}\right]$. Тогда распределение $S_{n}^{*}$ слабо сходится к стандартному нормальному закону, причем

$$
\sup _{x}\left|F_{N}(x)-\Phi(x)\right| \leqslant c \frac{\beta^{*}}{\sigma^{*} \sqrt{N}}
$$

где $\Phi(x)$ - функция распределения стандартного нормального закона и

$$
\sigma^{*}=\min _{\theta_{0} \leqslant \theta \leqslant \theta_{1}} \sigma(\theta)>0, \quad \beta^{*}=\max _{\theta_{0} \leqslant \theta \leqslant \theta_{1}} \beta(\theta),
$$

c- постоянная из неравенства Берри-Эссеена.

Доказательство. Справедливость теоремы следует из того, что если зафиксировать произвольное $\theta$, то по теореме Берри-Эссеена

$$
\sup \left|F_{N}(x)-\Phi(x)\right| \leqslant c \frac{\beta(\theta)}{\sigma(\theta) \sqrt{N}} \leqslant c \frac{\beta^{*}}{\sigma^{*} \sqrt{N}} .
$$

Положительность $\sigma^{*}$ следует из ограничений, наложенных на последовательность $b_{0}, b_{1}, \ldots$

Теорема 2. Пусть $N \rightarrow \infty$ и параметр $\theta=\theta(N) \rightarrow 0$ так, что выполняется соотношение

$$
\frac{b_{1}}{b_{0}} N \theta \rightarrow \lambda,
$$

где $\lambda$ - некоторая положительная постоянная. Тогда распределение суммы $S_{N}$ сходится $\kappa$ распределению Пуассона с параметром $\lambda$. 
Доказательство. Применим теорему непрерывности для производящих функций (см., например, [5]). Производящая функщия распределения Пуассона с параметром $\lambda$ есть $\phi_{\Pi}(z)=e^{-\lambda(1-z)}$, в то время как производящая функщия распределения случайной величины $\xi$ есть $\phi(z)=B(z \theta) / B(\theta)$. Производящая функция суммы $S_{N}$ есть, очевидно, $\phi^{N}(z)$. Пользуясь асимптотическим разложением $\ln (1+x)=x+O\left(x^{2}\right)$, справедливым при $|x|<1$, получаем, что при $N \rightarrow \infty, \theta \rightarrow 0$ на множестве $0<z<1$

$$
\begin{aligned}
\ln \phi^{N}(z)= & N \ln \phi(z)=N(\ln B(\theta z)-\ln B(\theta)) \\
= & N \ln \left(b_{0}+b_{1} \theta z+O\left(\theta^{2}\right)\right)-N \ln \left(b_{0}+b_{1} \theta+O\left(\theta^{2}\right)\right) \\
= & N \ln \left(1+\frac{b_{1}}{b_{0}} \theta z+O\left(\theta^{2}\right)\right)-N \ln \left(1+\frac{b_{1}}{b_{0}} \theta+O\left(\theta^{2}\right)\right) \\
= & \frac{b_{1}}{b_{0}} N \theta(z-1)+O\left(N \theta^{2}\right)=\lambda(z-1)(1+o(1)),
\end{aligned}
$$

что доказывает сходимость распределения суммы $S_{N}$ к распределению Пуассона с параметром $\lambda$.

Теорема 3. Пусть $N \rightarrow \infty$ и параметр $\theta=\theta(N) \rightarrow 0$ так, что $\theta N \rightarrow \infty$. Тогда распределение суммы $S_{N}^{*}$ слабо сходится к стандартному нормальному закону.

Доказательство. Представим $B\left(\theta e^{i t /(\sigma \sqrt{N})}\right)$ в виде $B\left(\theta e^{i t /(\sigma \sqrt{N})}\right)=B(\theta+\Delta \theta)$, где $\Delta=e^{i t /(\sigma \sqrt{N})}-1$. Возьмем логарифм от обеих частей представления характеристической функции (3). Разлагая $\ln B(\theta+\Delta \theta)$ в точке $\theta$ до членов третьего порядка малости при фиксированном $t$, получаем, что

$$
\ln B(\theta+\Delta \theta)=\ln \left(B(\theta)+\Delta \theta B^{\prime}(\theta)+\frac{(\Delta \theta)^{2}}{2} B^{\prime \prime}(\theta)+O\left(\Delta^{3} \theta^{3}\right)\right),
$$

так как производная $B^{\prime \prime \prime}(\theta)$ существует и ограничена при ограниченном $\theta \leqslant \theta_{1}<R$. Далее, пользуясь разложением $\ln (1+x)=x-x^{2} / 2+O\left(x^{3}\right)$ при $x \rightarrow 0$, получаем, что

$$
\begin{aligned}
\ln B(\theta+\Delta \theta) & =\ln B(\theta)+\ln \left(1+\frac{\Delta \theta B^{\prime}(\theta)}{B(\theta)}+\frac{\Delta^{2} \theta^{2} B^{\prime \prime}(\theta)}{2 B(\theta)}+O\left(\Delta^{3} \theta^{3}\right)\right) \\
& =\ln B(\theta)+\frac{\Delta \theta B^{\prime}(\theta)}{B(\theta)}+\frac{\Delta^{2} \theta^{2} B^{\prime \prime}(\theta)}{2 B(\theta)}-\frac{\Delta^{2} \theta^{2}\left(B^{\prime}(\theta)\right)^{2}}{2(B(\theta))^{2}}+O\left(\Delta^{3} \theta^{3}\right) .
\end{aligned}
$$

Таким образом,

$$
\begin{aligned}
\ln \varphi^{*}(t) & =\ln \frac{B\left(\theta e^{i t /(\sigma \sqrt{N})}\right)}{B(\theta)} e^{-i t m /(\sigma \sqrt{N})} \\
& =\ln B(\theta+\Delta \theta)-\ln B(\theta)-i t m /(\sigma \sqrt{N}) \\
& =\frac{\Delta \theta B^{\prime}(\theta)}{B(\theta)}+\frac{\Delta^{2} \theta^{2} B^{\prime \prime}(\theta)}{2 B(\theta)}-\frac{\Delta^{2} \theta^{2}\left(B^{\prime}(\theta)\right)^{2}}{2(B(\theta))^{2}}-\frac{i t m}{\sigma \sqrt{N}}+O\left(\Delta^{3} \theta^{3}\right) .
\end{aligned}
$$

Легко видеть, что при $\theta \rightarrow 0$, в силу ранее полученных выражений для $m(\theta)$ и $\sigma^{2}(\theta)$, справедливы представления

$$
\begin{array}{r}
m=m(\theta)=\mathbf{M} \xi=\theta \frac{B^{\prime}(\theta)}{B(\theta)}=\theta \frac{b_{1}}{b_{0}}+O\left(\theta^{2}\right), \\
\sigma^{2}=\sigma^{2}(\theta)=\mathbf{D} \xi=\theta \frac{b_{1}}{b_{0}}+O\left(\theta^{2}\right) .
\end{array}
$$


Далее, очевидно,

$$
\Delta \frac{\theta B^{\prime}(\theta)}{B(\theta)}-\frac{i t m}{\sigma \sqrt{N}}=\Delta m-\frac{i t m}{\sigma \sqrt{N}} .
$$

При любом фиксированном $t$ при $N \rightarrow \infty$

$$
\Delta=e^{i t /(\sigma \sqrt{N})}-1=\frac{i t}{\sigma \sqrt{N}}-\frac{t^{2}}{2 \sigma^{2} N}+O\left(1 /\left(\sigma^{3} N^{3 / 2}\right)\right)
$$

откуда следует, что

$$
\Delta m-\frac{i t m}{\sigma \sqrt{N}}=-\frac{t^{2} m}{2 \sigma^{2} N}+O\left(1 /\left(\sigma^{1 / 2} N^{3 / 2}\right)\right)
$$

Окончательно получаем следующую оценку для логарифма характеристической функщии случайной величины $\xi$ :

$$
\begin{aligned}
\ln \varphi^{*}(t) & =-\frac{t^{2} m}{2 \sigma^{2} N}+\frac{\Delta^{2}}{2}\left(\frac{\theta^{2} B^{\prime \prime}(\theta)}{B(\theta)}-\frac{\left(\theta B^{\prime}(\theta)\right)^{2}}{(B(\theta))^{2}}\right)+O\left(1 /\left(\theta^{1 / 2} N^{3 / 2}\right)\right) \\
& =-\frac{t^{2} m}{2 \sigma^{2} N}-\frac{t^{2}}{2 \sigma^{2} N}\left(\mathbf{M} \xi(\xi-1)-(\mathbf{M} \xi)^{2}\right)+O\left(1 /\left(\theta^{1 / 2} N^{3 / 2}\right)\right) \\
& =-\frac{t^{2}}{2 \sigma^{2} N}\left(\mathbf{M} \xi+\mathbf{M} \xi(\xi-1)-(\mathbf{M} \xi)^{2}\right)+O\left(1 /\left(\theta^{1 / 2} N^{3 / 2}\right)\right) \\
& =-\frac{t^{2}}{2 N}+O\left(1 /\left(\theta^{1 / 2} N^{3 / 2}\right)\right) .
\end{aligned}
$$

Поэтому

$$
\left(\varphi^{*}(t)\right)^{N}=e^{-t^{2} / 2}(1+O(1 / \sqrt{\theta N}))
$$

что завершает доказательство теоремы

\section{4. Локальные предельные теоремы}

Как уже отмечалось во введении, при применении обобщенной схемы размещения особое значение имеют локальные предельные теоремы. В этом разделе доказаны локальные предельные теоремы в случае, когда параметр $\theta=\theta(N)$ при $N \rightarrow \infty$ изменяется так, что $\theta \leqslant \theta_{1}<R$.

Теорема 4. Пусть $N \rightarrow \infty$ и существуют положительные постоянные $\theta_{0}, \theta_{1}$, такие, что параметр $\theta=\theta(N)$ меняется так, что $\theta_{0} \leqslant \theta \leqslant \theta_{1}<R$. Тогда

$$
\sigma \sqrt{N} P_{N}(n)-\frac{1}{\sqrt{2 \pi}} \exp \left\{-\frac{(n-m N)^{2}}{2 \sigma^{2} N}\right\} \rightarrow 0
$$

равномерно относительно чельх неотричательных $n$.

Доказательство. Равномерная по $n$ справедливость соотношения (5) при любом фиксированном $\theta$ установлена в $\S 49$ в [15]. 
Покажем, что указанное соотношение выполняется равномерно по $\theta$. Будем следовать доказательству локальной предельной теоремы, предложенному Б. В. Гнеденко в $\$ 49$ в [15]; при этом будем следить за равномерностью оценок остаточных членов.

Характеристическая функция суммы $S_{N}$ есть $\varphi^{N}(t)$. По теореме обращения для характеристических функций

$$
P_{N}(n)=\frac{1}{2 \pi} \int_{-\pi}^{\pi} \varphi^{N}(t) e^{-i t n} d t
$$

Положим

$$
n=m N+\sigma z \sqrt{N}
$$

Тогда, очевидно,

$$
P_{N}(n)=\frac{1}{2 \pi} \int_{-\pi}^{\pi} e^{-i t \sigma z \sqrt{N}} e^{-i t m N} \varphi^{N}(t) d t=\frac{1}{2 \pi} \int_{-\pi}^{\pi} e^{-i t \sigma z \sqrt{N}}(\bar{\varphi}(t))^{N} d t .
$$

Поскольку

$$
\frac{1}{\sqrt{2 \pi}} e^{-z^{2} / 2}=\frac{1}{2 \pi} \int_{-\infty}^{\infty} e^{-i x z} e^{-x^{2} / 2} d x
$$

разность

$$
R_{N}=2 \pi\left(\sigma \sqrt{N} P_{N}(n)-\frac{1}{\sqrt{2 \pi}} e^{-z^{2} / 2}\right)
$$

можно представить в виде суммы следующих четырех интегралов:

$$
\begin{aligned}
& I_{1}=\int_{-A}^{A} e^{-i x z}\left((\bar{\varphi}(x /(\sigma \sqrt{N})))^{N}-e^{-x^{2} / 2}\right) d x, \\
& I_{2}=-\int_{|x| \geqslant A} e^{-i x z-x^{2} / 2} d x, \\
& I_{3}=\int_{A \leqslant|x| \leqslant \varepsilon \sigma \sqrt{N}} e^{-i x z}(\bar{\varphi}(x /(\sigma \sqrt{N})))^{N} d x \\
& I_{4}=\int_{\varepsilon \sigma \sqrt{N} \leqslant|x| \leqslant \pi \sigma \sqrt{N}} e^{-i x z}(\bar{\varphi}(x /(\sigma \sqrt{N})))^{N} d x ;
\end{aligned}
$$

положительные постоянные $A$ и $\varepsilon$ будут выбраны позднее.

Возьмем произвольное $\delta>0$ и покажем, что разность $R_{N}$ может быть сделана меньше $\delta$ равномерно по $n$ и $\theta, \theta_{0} \leqslant \theta \leqslant \theta_{1}$, выбором достаточно большого $N$.

Интеграл $I_{2}$ не зависит от $\theta$,

$$
\left|I_{2}\right| \leqslant \int_{|x| \geqslant A} e^{-x^{2} / 2} d x,
$$

и этот интеграл может быть сделан сколь угодно малым для всех $n$ и $\theta, \theta_{0} \leqslant \theta \leqslant \theta_{1}$, выбором достаточно большого $A$.

Поскольку третья производная $B^{\prime \prime \prime}(z)$ ограничена при $|z| \leqslant \theta_{1}<R$, справедливо представление

$$
\bar{\varphi}(t)=1-\frac{\sigma^{2} t^{2}}{2}+c(\theta, t) t^{3}
$$


где $|c(\theta, t)| \leqslant c$ при всех $n$ и $\theta, \theta_{0} \leqslant \theta \leqslant \theta_{1}$. Отсюда следует, что существует $\varepsilon>0$ такое, что при $|t| \leqslant \varepsilon$

$$
|\bar{\varphi}(t)| \leqslant 1-\frac{\sigma^{2} t^{2}}{4} \leqslant e^{-\sigma^{2} t^{2} / 4}
$$

Поэтому

$$
\left|I_{3}\right| \leqslant \int_{A \leqslant|x| \leqslant \varepsilon \sigma \sqrt{N}}(\bar{\varphi}(x /(\sigma \sqrt{N})))^{N} d x \leqslant \int_{A \leqslant|x| \leqslant \varepsilon \sigma \sqrt{N}} e^{-x^{2} / 4} d x,
$$

и этот интеграл может быть сделан сколь угодно малым для всех $n$ и $\theta, \theta_{0} \leqslant \theta \leqslant \theta_{1}$, выбором достаточно большого $A$.

Пусть $\varepsilon$ выбрано так, что выполнено (6), а $A$ так, что $\left|I_{2}\right| \leqslant \delta / 4,\left|I_{3}\right| \leqslant \delta / 4$. При так выбранных и зафиксированных $\varepsilon$ и $A$ оценим интегралы $I_{1}$ и $I_{4}$.

При условиях доказываемой теоремы справедлива теорема 1 , в силу которой распределение случайной величины $S_{N}^{*}$ слабо сходится к стандартному нормальному закону, и следовательно, при $N \rightarrow \infty$

$$
(\bar{\varphi}(x /(\sigma \sqrt{N})))^{N}-e^{-x^{2} / 2} \rightarrow 0
$$

равномерно по $x$ в любом конечном интервале. Более того, эта сходимость равномерна по $\theta, \theta_{0} \leqslant \theta \leqslant \theta_{1}<R$ (если предположить противное, то приходим к противоречию с теоремой 1). Следовательно, найдется такое $N_{0}$, что при $N \geqslant N_{0}$

$$
\left|(\bar{\varphi}(x /(\sigma \sqrt{N})))^{N}-e^{-x^{2} / 2}\right| \leqslant \delta /(8 A)
$$

для всех $x,-A \leqslant x \leqslant A$, и для всех $\theta, \theta_{0} \leqslant \theta \leqslant \theta_{1}$. Следовательно, $\left|I_{1}\right| \leqslant \delta / 4$ при достаточно большом $N$.

Наконец, справедлива оценка

$$
\left|I_{4}\right| \leqslant \int_{\varepsilon \sigma \sqrt{N} \leqslant|x| \leqslant \pi \sigma \sqrt{N}}|\bar{\varphi}(x /(\sigma \sqrt{N}))|^{N} d x=\sigma \sqrt{N} \int_{\varepsilon \leqslant|t| \leqslant \pi}|\varphi(t)|^{N} d t .
$$

При любом $\theta$ шаг распределения случайной величины $\xi$ равен единице, поэтому

$$
\max _{\varepsilon \leqslant|t| \leqslant \pi}|\varphi(t)|=q(\theta)<1
$$

Нетрудно видеть, что $q(\theta)$ непрерывно зависит от параметра $\theta$ на отрезке $\left[\theta_{0}, \theta_{1}\right]$ и, следовательно, достигает своего максимума в некоторой точке $\bar{\theta}$ этого отрезка. Таким образом,

$$
\max _{\theta \in\left\{\theta_{0}, \theta_{1} \mid\right.} \max _{\varepsilon \leqslant|t| \leqslant \pi}|\varphi(t)|=q(\bar{\theta})=q<1
$$

Поэтому

$$
\left|I_{4}\right| \leqslant 2 \pi \sigma \sqrt{N} q^{N}
$$

и выбором достаточно большого $N$ этот интеграл может быть сделан по модулю меньшим $\delta / 4$ равномерно по $\theta, \theta_{0} \leqslant \theta \leqslant \theta_{1}$. Теорема тем самым доказана. 
Теорема 5. Пусть $N \rightarrow \infty, \theta=\theta(N) \rightarrow 0, \theta N \rightarrow \infty$. Тогда

$$
\sigma \sqrt{N} P_{N}(n)-\frac{1}{\sqrt{2 \pi}} \exp \left\{-\frac{(n-m N)^{2}}{2 \sigma^{2} N}\right\} \rightarrow 0
$$

равномерно относительно челых неотричательных $n$.

Доказательство. Оценим опять интегралы $I_{1}-I_{4}$, введенные в доказательстве теоремы 4 , в условиях настоящей теоремы. Интеграл $I_{2}$, не зависящий от $\theta$, оценивается как в доказательстве теоремы 4 и может быть сделан сколь угодно малым выбором достаточно большого $A$. Также выбором достаточно большого $A$ и достаточно малого $\varepsilon$ сколь угодно малым может быть сделан интеграл $I_{3}$. При так выбранных и зафиксированных $A$ и $\varepsilon$, в силу теоремы 3 получаем, что интеграл $I_{1}$ также может быть сделан сколь угодно малым выбором достаточно большого $N$. Остается оценить интеграл $I_{4}$. Справедлива оценка

$$
\left|I_{4}\right| \leqslant \sigma \sqrt{N} \int_{\varepsilon \leqslant|t| \leqslant \pi}|\varphi(t)|^{N} d t .
$$

Напомним, что

$$
\varphi(t)=\frac{B\left(\theta e^{i t}\right)}{B(\theta)} .
$$

Оценим $\varphi(t)$ при $\varepsilon \leqslant|t| \leqslant \pi$. При $\theta \rightarrow 0$ справедливо разложение

$$
B\left(\theta e^{i t}\right)=B\left(\theta+\theta\left(e^{i t}-1\right)\right)=B(\theta)+\theta\left(e^{i t}-1\right) B^{\prime}(\theta)+O\left(\theta^{2}\right) .
$$

Тогда

$$
\begin{aligned}
\ln \varphi(t) & =\ln B\left(\theta e^{i t}\right)-\ln B(\theta) \\
& =\ln B(\theta)+\ln \left(1+\theta\left(e^{i t}-1\right)\left(B^{\prime}(\theta) / B(\theta)\right)+O\left(\theta^{2}\right)\right)-\ln B(\theta) \\
& =\theta\left(e^{i t}-1\right)\left(B^{\prime}(\theta) / B(\theta)\right)+O\left(\theta^{2}\right)
\end{aligned}
$$

Так как $e^{i t}-1=\cos t+i \sin t-1$, получаем, что

$$
\varphi(t)=\exp \left\{-\theta(1-\cos t)\left(B^{\prime}(\theta) / B(\theta)\right)+O\left(\theta^{2}\right)\right\} \exp \left\{i \theta \sin t\left(B^{\prime}(\theta) / B(\theta)\right)\right\} .
$$

Поскольку $B^{\prime}(\theta) / B(\theta)=b_{1} / b_{0}+O(\theta)$ при $\theta \rightarrow 0$, при $\varepsilon \leqslant|t| \leqslant \pi, \varepsilon<\pi / 2$, при достаточно малых $\theta$

$$
|\varphi(t)| \leqslant e^{-\theta b_{1}(1-\cos \varepsilon) /\left(2 b_{0}\right)} .
$$

Тогда

$$
\left|I_{4}\right| \leqslant \sigma \sqrt{N} 2 \pi e^{-\theta b_{1} N(1-\cos \varepsilon) /\left(2 b_{0}\right)}
$$

и, учитывая, что при $\theta \rightarrow 0$ и $\theta N \rightarrow \infty$

$$
\sigma^{2}=\frac{b_{1}}{b_{0}} \theta+O\left(\theta^{2}\right)
$$

получаем, что интеграл $I_{4}$ может быть сделан сколь угодно малым.

Теорема тем самым доказана. 


\section{Список литературы}

1. В. Ф. Колчин, Один класс предельных теорем для условных распределений. Литовский матем. сборник (1968) 8, №1, 111-126.

2. В. Ф. Колчин, Б. А. Севастьянов, В. П. Чистяков, Случайные размещения. Наука, Москва, 1976.

3. В. Ф. Колчин, Случайные отображения. Наука, Москва, 1984.

4. В. Ф. Колчин, Случайные графы. Физматлит, Москва, 2000.

5. В. Ф. Колчин, Асимптотические методы теории вероятностей. МИЭМ, Москва, 1984.

6. Yu. L. Pavlov, Random forests. VSP, Utrecht, 2000.

7. Н. И. Казимиров, Ю. Л. Павлов, Одно замечание о лесах Гальтона-Ватсона. Дискретная математика (2000) 12, №1, 47-59.

8. Н. И. Казимиров, О некоторых условиях отсутствия гигантской компоненты в обобщенной схеме размещения. Дискретная математика (2002) 14, №2, 107-118.

9. T. B. Myllari, Limit distributions of the number of leaves of a Galton-Watson forest. In: Probabilistic Methods in Discrete Mathematics. VSP, Utrecht, 2002, pp. 257-271.

10. А. Б. Мухин, Локальные предельные теоремы для решетчатых случайных величин. Теория вероятностей и ее применения (1991) 36, №4, 660-674.

11. A. V. Kolchin, On the number of hyperforests. J. Math. Sci. (1995) 76, 2250-2258.

12. A. V. Kolchin, On the asymptotic behaviour of the number of hyperforests. In: Probabilistic Methods in Discrete Mathematics. VSP, Utrecht, 1996, pp. 285-294.

13. А. Н. Тимашёв, Об асимптотических разложениях в локальных предельных теоремах в равновероятных схемах размещения частиц по различным ячейкам. Дискретная математика (2000) 12, №1, 60-69.

14. А. Н. Тимашёв, О распределении числа циклов заданной длины в классе подстановок с известным числом циклов. Дискретная математика (2001) 13, №4, 60-72.

15. Б. В. Гнеденко, А. Н. Колмогоров, Предельные распределения для сумм независимых случайных величин. ГИТТЛ, Москва-Ленинград, 1949.

Статья поступила 13.10.2003. 